doi: $10.2306 /$ scienceasia1513-1874.2014.40.182

\title{
Bounds of the normal approximation to random-sum Wilcoxon statistics
}

\author{
Mongkhon Tuntapthai, Nattakarn Chaidee* \\ Department of Mathematics and Computer Science, Faculty of Science, Chulalongkorn University, \\ Bangkok 10330 Thailand
}

${ }^{*}$ Corresponding author, e-mail: nattakarn.c@chula.ac.th.

\begin{abstract}
Consider sequences $\left\{X_{i}\right\}_{i=1}^{\infty}$ and $\left\{Y_{j}\right\}_{j=1}^{\infty}$ of independent and identically distributed (i.i.d.) random variables, random variables $K_{1}, K_{2}$ ranging over of all positive integers, where the $X_{i}$ 's, $Y_{j}$ 's, $K_{1}$, and $K_{2}$ are all independent. We obtain Berry-Esseen bounds for random-sum Wilcoxon's statistics in the form $\left(W_{K_{1}, K_{2}}-U\right) / V$ and $\left(W_{K_{1}, K_{2}}-a\right) / b$ where $W_{K_{1}, K_{2}}=\sum_{i=1}^{K_{1}} \sum_{j=1}^{K_{2}} I\left(X_{i}>Y_{j}\right)$ and $U, V$ are random variables, and $a, b$ are constants. We also show that the rate of convergence is $O\left(\left(E K_{2}\right)^{-1 / 2}\right)$ provided by $E K_{1} / E K_{2} \rightarrow \tau$ for some constant $\tau>0$ when $E K_{1}$ and $E K_{2}$ tend to infinity.
\end{abstract}

KEYWORDS: Wilcoxon's rank-sum statistics, Mann-Whitney's statistics, random-indexed summands, BerryEsseen bounds, non-random centring

\section{INTRODUCTION}

Let $X, X_{1}, \ldots, X_{m}$ and $Y, Y_{1}, \ldots, Y_{n}$ be two sequences of independent and identically distributed (i.i.d.) random variables. Note that the distributions of $X$ and $Y$ are not necessarily identical. Suppose the $X_{i}$ 's and $Y_{j}$ 's are independent and continuous random variables. To test the null hypothesis that the distributions of $X$ and $Y$ are equal, Wilcoxon ${ }^{1}$ introduced rank-sum statistics by ranking the random variables between two sequences and then computing the sum of these rankings. Mann and Whitney ${ }^{2}$ proposed the Mann-Whitney's statistic,

$$
W_{m, n}=\sum_{i=1}^{m} \sum_{j=1}^{n} I\left(X_{i}>Y_{j}\right) \text {. }
$$

Mann and Whitney ${ }^{2}$ showed that their statistics and Wilcoxon's rank-sum statistics are equivalent. They also showed that under the null hypothesis, MannWhitney's distribution can be approximated by the standard normal distribution denoted by $\Phi$.

Consider a Wilcoxon's rank-sum statistic defined by $\mathcal{W}_{m}=\sum_{k=1}^{m} R_{k}$ where $R_{k}$ is the ranking-number of $X_{k}$ between two samples $X_{1}, \ldots, X_{m}$ and $Y_{1}$, $\ldots, Y_{n}$. Pestman ${ }^{3}$ obtained normal approximation theorems and Alberink ${ }^{4}$ investigated Berry-Esseen bound for Wilcoxon's rank-sum statistics by assuming the null hypothesis. From the result of Alberink ${ }^{4}$ and the well-known fact that $\mathcal{W}_{m}=W_{m, n}+m(m+1) / 2$, we obtain the bound for Mann-Whitney's statistics as the next statement.

Theorem 1 (Ref. 4) Under the null hypothesis that the distributions of $X$ and $Y$ are equal, we have the following result:

$$
\begin{array}{r}
\sup _{x \in \mathbb{R}}\left|P\left(\frac{W_{m, n}-m n / 2}{\sqrt{m n(m+n) / 12}} \leqslant x\right)-\Phi(x)\right| \\
\leqslant \frac{17}{\sqrt{m+n}}\left\{1+\frac{3 \sqrt{3}\left(m^{2}+n^{2}\right)}{4 \sqrt{m n}(m+n)}\right\} .
\end{array}
$$

It is obvious that Mann-Whitney's statistics are example of $U$-statistics introduced by Hoeffding ${ }^{5}$ and Lehmann $^{6}$. So that the bounds for Mann-Whitney's statistics were established by applying the results of Grams and Serfling ${ }^{7}$ or Chen and $\mathrm{Shao}^{8}$. Now, we need some notation:

$$
\begin{aligned}
\theta & =P(X>Y), \\
\mu & =\theta(1-\theta), \\
\sigma_{1}^{2} & =E|E\{I(X>Y) \mid X\}-\theta|^{2}, \\
\gamma_{1} & =E|E\{I(X>Y) \mid X\}-\theta|^{3}, \\
\sigma_{2}^{2} & =E|E\{I(X>Y) \mid Y\}-\theta|^{2}, \\
\gamma_{2} & =E|E\{I(X>Y) \mid Y\}-\theta|^{3} .
\end{aligned}
$$


Consider a two-sample $U$-statistic given by

$$
U_{m, n}=\frac{1}{m} \frac{1}{n} \sum_{i=1}^{m} \sum_{j=1}^{m} h\left(X_{i}, Y_{j}\right)
$$

where $h\left(X_{i}, Y_{j}\right)=I\left(X_{i}>Y_{j}\right)-\theta$. Observe that $E h(X, Y)=0, E h^{2}(X, Y)=\mu$ and that $\sigma_{\ell}^{2} \leqslant \mu$ for all $\ell=1,2$. Also, we note that

$$
\frac{U_{m, n}}{\sqrt{\sigma_{1}^{2} / m+\sigma_{2}^{2} / n}}=\frac{W_{m, n}-m n \theta}{\sqrt{m n^{2} \sigma_{1}^{2}+n m^{2} \sigma_{2}^{2}}} .
$$

Hence we can use the bounds for $U_{m, n}$ of Chen and $\mathrm{Shao}^{8}$ to derive the bounds for Mann-Whitney's statistics as the next statement.

\section{Theorem 2 (Ref. 8)}

$$
\begin{array}{r}
\sup _{x \in \mathbb{R}}\left|P\left(\frac{W_{m, n}-m n \theta}{\sqrt{m n\left(n \sigma_{1}^{2}+m \sigma_{2}^{2}\right)}} \leqslant x\right)-\Phi(x)\right| \\
\leqslant \frac{(1+\sqrt{2}) \sqrt{\mu}(1 / m+1 / n)}{\sqrt{\sigma_{1}^{2} / m+\sigma_{2}^{2} / n}} \\
+\frac{6.6\left(\gamma_{1} / m^{2}+\gamma_{2} / n^{2}\right)}{\left(\sigma_{1}^{2} / m+\sigma_{2}^{2} / n\right)^{3 / 2}},
\end{array}
$$

and there exists a constant $C$ (which does not depend on $m$ and $n$ ) such that for all $x \in \mathbb{R}$,

$$
\begin{gathered}
\left|P\left(\frac{W_{m, n}-m n \theta}{\sqrt{m n\left(n \sigma_{1}^{2}+m \sigma_{2}^{2}\right)}} \leqslant x\right)-\Phi(x)\right| \\
\leqslant \frac{9 \mu(1 / m+1 / n)^{2}}{(1+x)^{2}\left(\sigma_{1}^{2} / m+\sigma_{2}^{2} / n\right)} \\
+\frac{13.5 \sqrt{\mu}(1 / m+1 / n)}{\mathrm{e}^{x / 3} \sqrt{\sigma_{1}^{2} / m+\sigma_{2}^{2} / n}} \\
+\frac{C\left(\gamma_{1} / m^{2}+\gamma_{2} / n^{2}\right)}{(1+x)^{3}\left(\sigma_{1}^{2} / m+\sigma_{2}^{2} / n\right)^{3 / 2}} .
\end{gathered}
$$

In particular, if $\theta=1 / 2$ and $\sigma_{1}^{2}=\sigma_{2}^{2}=1 / 12$, then the constant 17 in Theorem 1 can be replaced by $(1+$ $\sqrt{2}) \sqrt{3}$.

Mann-Whitney's and Wilcoxon's statistics are usually used to compare two treatments in many comparative experiments. However, these statistics cannot be used when the sample sizes of observation from two treatments are not measured ${ }^{9,10}$. For example, the number of normal and abnormal images in the medical imaging studies and diagnostic marker studies. In 2011, under the situation that the sample sizes are random variables, Tang and Balakrishnan ${ }^{10}$ proposed a random-sum Wilcoxon's statistic as the following:
Let $\left\{X_{i}\right\}_{i=1}^{\infty}$ and $\left\{Y_{j}\right\}_{j=1}^{\infty}$ be two sequences of i.i.d. random variables such that the $X_{i}$ 's and $Y_{j}$ 's are independent. Assume that the $X_{i}$ 's and $Y_{j}$ 's are continuous random variables. Let $K_{1}$ and $K_{2}$ be independent positive integer-valued random variables which are independent of the $X_{i}$ 's and $Y_{j}$ 's. $A$ random-sum Wilcoxon's statistic is defined by

$$
W_{K_{1}, K_{2}}=\sum_{i=1}^{K_{1}} \sum_{j=1}^{K_{2}} I\left(X_{i}>Y_{j}\right) .
$$

Tang and Balakrishnan ${ }^{10}$ also obtained normal approximation theorems for random-sum Wilcoxon's statistics as the next statement.

Theorem 3 (Ref. 10) For each $\ell=1,2$, let $K_{\ell}$ be a positive integer-valued random variable depending on a parameter $\tau_{\ell}$. Suppose that

(C1) the random variable $K_{\ell}$ has its asymptotic distribution as the normal distribution with mean $E K_{\ell}$ and variance $\operatorname{Var}\left(K_{\ell}\right)$, as $\tau_{\ell} \rightarrow \infty$;

(C2) $E K_{2} / E K_{1} \rightarrow \eta$, for some finite non-zero constant $\eta$, as $\tau_{\ell} \rightarrow \infty$;

(C3) $\operatorname{Var}\left(K_{\ell}\right) / E K_{\ell} \rightarrow \delta_{\ell}$, for some finite constant $\delta_{\ell}$ as $\tau_{\ell} \rightarrow \infty$;

(C4) $\left(E K_{\ell}\right)^{p} / \tau_{\ell} \rightarrow \lambda_{\ell}$, for some finite non-zero constant $\lambda_{\ell}$ and for some $p \geqslant 1$, as $\tau_{\ell} \rightarrow \infty$.

Then

$\left|P\left(\frac{\tau_{1}^{-3 / 2 p}\left(W_{K_{1}, K_{2}}-\theta E K_{1} E K_{2}\right)}{\sqrt{\operatorname{Var}\left(\tau_{1}^{-3 / 2 p} W_{K_{1}, K_{2}}\right)}} \leqslant x\right)-\Phi(x)\right|$

tends to zero, where

$$
\begin{aligned}
\operatorname{Var} & \left(\tau_{1}^{-3 / 2 p} W_{K_{1}, K_{2}}\right) \\
& \rightarrow \lambda_{1}^{3} \eta^{2} \sigma_{1}^{2}+\lambda_{1}^{3} \eta \sigma_{2}^{2}+\left(\delta_{1} \lambda_{1}^{3} \eta^{2}+\delta_{2} \lambda_{1}^{3} \eta\right) \theta^{2}, \\
\text { as } \tau_{1} & \rightarrow \infty \text { and } \tau_{2} \rightarrow \infty .
\end{aligned}
$$

\section{MAIN RESULTS}

The main results of this article are Berry-Esseen bounds for random-sum Wilcoxon's statistics. In Theorem 4, we consider a random-sum Wilcoxon's statistic with random centring and random norming as in the form $\left(W_{K_{1}, K_{2}}-U\right) / V$ where $U$ and $V$ are random variables. In Theorem 5, we consider a random-sum Wilcoxon's statistic with non-random centring and non-random norming as in the form $\left(W_{K_{1}, K_{2}}-a\right) / b$ where $a$ and $b$ are constants. 
Theorem 4 Let $K_{1}$ and $K_{2}$ be positive integer-valued random variables such that the $X_{i}$ 's, $Y_{j}$ 's, $K_{1}, K_{2}$ are independent. We have the following results:

$$
\begin{gathered}
\sup _{x \in \mathbb{R}}\left|P\left(\frac{W_{K_{1}, K_{2}}-\theta K_{1} K_{2}}{\sqrt{\sigma_{1}^{2} K_{1} K_{2}^{2}+\sigma_{2}^{2} K_{2} K_{1}^{2}}} \leqslant x\right)-\Phi(x)\right| \\
\leqslant \frac{11.2}{\sqrt{E K_{2}}}\left\{\sqrt{\frac{\operatorname{Var}\left(K_{1}\right)}{E K_{1}}} \sqrt{\frac{E K_{2}}{E K_{1}}}+\sqrt{\frac{\operatorname{Var}\left(K_{2}\right)}{E K_{2}}}\right\} \\
+\frac{3.1 \sqrt{\mu}\left(\sqrt{E K_{2} / E K_{1}}+\sqrt{E K_{1} / E K_{2}}\right)}{\sqrt{E K_{2}} \sqrt{\sigma_{1}^{2}+\sigma_{2}^{2} E K_{1} / E K_{2}}} \\
+\frac{11.9\left\{\gamma_{1} \sqrt{E K_{2} / E K_{1}}+\gamma_{2}\left(E K_{1} / E K_{2}\right)^{3 / 2}\right\}}{\sqrt{E K_{2}}\left(\sigma_{1}^{2}+\sigma_{2}^{2} E K_{1} / E K_{2}\right)^{3 / 2}},
\end{gathered}
$$

and there exists a constant $C$ such that for all $x \in \mathbb{R}$,

$$
\begin{gathered}
\mid P\left(\frac{W_{K_{1}, K_{2}}-\theta K_{1} K_{2}}{\left.\sqrt{\sigma_{1}^{2} K_{1} K_{2}^{2}+\sigma_{2}^{2} K_{2} K_{1}^{2}} \leqslant x\right)-\Phi(x) \mid}\right. \\
\leqslant \frac{C \mu}{(1+x)^{2} E K_{2}}\left\{\frac{\operatorname{Var}\left(K_{1}\right)}{\sigma_{2}^{2} E K_{1}} \frac{E K_{2}}{E K_{1}}+\frac{\operatorname{Var}\left(K_{2}\right)}{\sigma_{1}^{2} E K_{2}}\right\} \\
+\frac{C \mu\left(\sqrt{E K_{2} / E K_{1}}+\sqrt{E K_{1} / E K_{2}}\right)^{2}}{(1+x)^{2} E K_{2}\left(\sigma_{1}^{2}+\sigma_{2}^{2} E K_{1} / E K_{2}\right)} \\
+\frac{C \sqrt{\mu}\left(\sqrt{E K_{2} / E K_{1}}+\sqrt{E K_{1} / E K_{2}}\right)}{(1+x)^{3} \sqrt{E K_{2}} \sqrt{\sigma_{1}^{2}+\sigma_{2}^{2} E K_{1} / E K_{2}}} \\
+\frac{C\left\{\gamma_{1} \sqrt{E K_{2} / E K_{1}}+\gamma_{2}\left(E K_{1} / E K_{2}\right)^{3 / 2}\right.}{(1+x)^{3} \sqrt{E K_{2}}\left(\sigma_{1}^{2}+\sigma_{2}^{2} E K_{1} / E K_{2}\right)^{3 / 2}} .
\end{gathered}
$$

Remark 1 If $K_{1}$ and $K_{2}$ satisfy the conditions (C2), (C3) and (C4) in Theorem 3, then the rate of convergence is $O\left(\tau_{2}^{-1 / 2 p}\right)$ for some $p \geqslant 1$, when $\tau_{1}$ and $\tau_{2}$ tend to infinity.

By definition, random-sum Wilcoxon's statistics include Mann-Whitney's statistics in the special case of fixed-indices $K_{1}=m$ and $K_{2}=n$. In this case, our results are same as Theorem 2 (the bounds of Chen and $\mathrm{Shao}^{8}$ ). But the constants in a uniform bound of Theorem 2 are smaller. The next corollary is an immediately consequence of Theorem 4 .

\section{Corollary 1}

$$
\begin{gathered}
\sup _{x \in \mathbb{R}}\left|P\left(\frac{W_{m, n}-m n \theta}{\sqrt{m n\left(n \sigma_{1}^{2}+m \sigma_{2}^{2}\right)}} \leqslant x\right)-\Phi(x)\right| \\
\leqslant \frac{3.1 \sqrt{\mu}}{\sqrt{n}} \frac{\sqrt{n / m}+\sqrt{m / n}}{\sqrt{\sigma_{1}^{2}+\sigma_{2}^{2} m / n}}
\end{gathered}
$$

$$
+\frac{11.9}{\sqrt{n}} \frac{\gamma_{1} \sqrt{n / m}+\gamma_{2}(n / m)^{3 / 2}}{\left(\sigma_{1}^{2}+\sigma_{2}^{2} m / n\right)^{3 / 2}},
$$

and there exists an absolute constant $C$ (which does not depend on $m$ and $n$ ) such that for all $x \in \mathbb{R}$,

$$
\begin{gathered}
\left|P\left(\frac{W_{m, n}-m n \theta}{\sqrt{m n\left(n \sigma_{1}^{2}+m \sigma_{2}^{2}\right)}} \leqslant x\right)-\Phi(x)\right| \\
\leqslant \frac{C \mu}{(1+x)^{2} n} \frac{(\sqrt{n / m}+\sqrt{m / n})^{2}}{\left(\sigma_{1}^{2}+\sigma_{2}^{2} m / n\right)} \\
+\frac{C \sqrt{\mu}}{(1+x)^{3} \sqrt{n}} \frac{\sqrt{n / m}+\sqrt{m / n}}{\sqrt{\sigma_{1}^{2}+\sigma_{2}^{2} m / n}} \\
+\frac{C}{(1+x)^{3} \sqrt{n}} \frac{\gamma_{1} \sqrt{n / m}+\gamma_{2}(n / m)^{3 / 2}}{\left(\sigma_{1}^{2}+\sigma_{2}^{2} m / n\right)^{3 / 2}} .
\end{gathered}
$$

It is understood that Wilcoxon's statistics can be approximated by the sum of independent random variables, introduced by Hajek ${ }^{11}$. Now, we denote the projection of $W_{K_{1}, K_{2}}$ on the $X_{i}$ 's, $Y_{j}$ 's, $K_{1}, K_{2}$, by

$$
\begin{aligned}
\widehat{W}_{K_{1}, K_{2}}:= & \sum_{i=1}^{K_{1}} E\left\{W_{K_{1}, K_{2}}-\theta E K_{1} E K_{2} \mid X_{i}\right\} \\
& +\sum_{j=1}^{K_{2}} E\left\{W_{K_{1}, K_{2}}-\theta E K_{1} E K_{2} \mid Y_{j}\right\} \\
& +E\left\{W_{K_{1}, K_{2}}-\theta E K_{1} E K_{2} \mid K_{1}, K_{2}\right\} \\
= & \left(E K_{2}\right) \sum_{i=1}^{K_{1}} E\left\{I\left(X_{i}>Y_{j}\right)-\theta \mid X_{i}\right\} \\
& +\left(E K_{1}\right) \sum_{j=1}^{K_{2}} E\left\{I\left(X_{i}>Y_{j}\right)-\theta \mid Y_{j}\right\} \\
& +\theta\left(K_{1} K_{2}-E K_{1} E K_{2}\right) .
\end{aligned}
$$

By taking the conditional expectation given by $K_{1}$ and $K_{2}$ (see p. 22 of Ref. 12), we observe that

$$
\begin{aligned}
& \operatorname{Var}\left(\widehat{W}_{K_{1}, K_{2}}\right) \\
&= E\left\{\operatorname{Var}\left(\widehat{W}_{K_{1}, K_{2}} \mid K_{1}, K_{2}\right)\right\} \\
&+\operatorname{Var}\left\{E\left(\widehat{W}_{K_{1}, K_{2}} \mid K_{1}, K_{2}\right)\right\} \\
&= \sigma_{1}^{2} E K_{1}\left(E K_{2}\right)^{2}+\sigma_{2}^{2} E K_{2}\left(E K_{1}\right)^{2} \\
&+\theta^{2}\left\{\left(E K_{2}\right)^{2} \operatorname{Var}\left(K_{1}\right)+\left(E K_{1}\right)^{2} \operatorname{Var}\left(K_{2}\right)\right\} \\
&+\theta^{2} \operatorname{Var}\left(K_{1}\right) \operatorname{Var}\left(K_{2}\right) .
\end{aligned}
$$

Set

$\operatorname{Var}^{*}\left(\widehat{W}_{K_{1}, K_{2}}\right):=\operatorname{Var}\left(\widehat{W}_{K_{1}, K_{2}}\right)-\theta^{2} \operatorname{Var}\left(K_{1}\right) \operatorname{Var}\left(K_{2}\right)$. 
Similarly, we can see that

$$
\begin{aligned}
\operatorname{Var} & \left(W_{K_{1}, K_{2}}\right) \\
= & E\left\{\operatorname{Var}\left(W_{K_{1}, K_{2}} \mid K_{1}, K_{2}\right)\right\} \\
& +\operatorname{Var}\left\{E\left(W_{K_{1}, K_{2}} \mid K_{1}, K_{2}\right)\right\} \\
= & E\left\{\mu K_{1} K_{2}\right\} \\
& +E\left\{\sigma_{1}^{2} K_{1} K_{2}\left(K_{2}-1\right)\right\} \\
& +E\left\{\sigma_{2}^{2} K_{1}\left(K_{1}-1\right) K_{2}\right\}+\operatorname{Var}\left(\theta K_{1} K_{2}\right) \\
= & \mu E K_{1} E K_{2} \\
& +\sigma_{1}^{2} E K_{1}\left\{\left(E K_{2}\right)^{2}+\operatorname{Var}\left(K_{2}\right)-E K_{2}\right\} \\
& +\sigma_{2}^{2} E K_{2}\left\{\left(E K_{1}\right)^{2}+\operatorname{Var}\left(K_{1}\right)-E K_{1}\right\} \\
& +\theta^{2}\left\{\left(E K_{2}\right)^{2} \operatorname{Var}\left(K_{1}\right)+\left(E K_{1}\right)^{2} \operatorname{Var}\left(K_{2}\right)\right\} \\
& +\theta^{2} \operatorname{Var}\left(K_{1}\right) \operatorname{Var}\left(K_{2}\right) .
\end{aligned}
$$

This implies that $\operatorname{Var}\left(W_{K_{1}, K_{2}}\right) / \operatorname{Var}^{*}\left(\widehat{W}_{K_{1}, K_{2}}\right) \rightarrow$ 1, when $E K_{1}$ and $E K_{2}$ tend to infinity. Hence, the quantity $\operatorname{Var}\left(W_{K_{1}, K_{2}}\right)$ for normalization in the normal approximation theorem, can be replaced by $\operatorname{Var}^{*}\left(\widehat{W}_{K_{1}, K_{2}}\right)$.

In the next statement, we show a uniform bound for random-sum Wilcoxon's statistics with nonrandom centring $\theta E K_{1} E K_{2}$ and non-random norming $\operatorname{Var}^{*}\left(\widehat{W}_{K_{1}, K_{2}}\right)$.

Theorem 5 Let $\xi_{1}, \ldots, \xi_{m}$ be i.i.d. positive integervalued random variables with $E\left(\xi_{1}\right)=a_{1}, \operatorname{Var}\left(\xi_{1}\right)=$ $b_{1}^{2}$ and $E\left|\xi_{1}\right|^{3}<\infty$. Let $\zeta_{1}, \ldots, \zeta_{n}$ be i.i.d. positive integer-valued random variables satisfying that $E\left(\zeta_{1}\right)=a_{2}, \operatorname{Var}\left(\zeta_{1}\right)=b_{2}^{2}$ and $E\left|\zeta_{1}\right|^{3}<\infty$. Suppose the $X_{i}$ 's, $Y_{j}$ 's, $\xi_{k}$ 's, $\zeta_{\ell}$ 's are independent. Put $K_{1}=\sum_{j=1}^{m} \xi_{j}, K_{2}=\sum_{\ell=1}^{n} \zeta_{\ell}$. Then

$$
\begin{aligned}
\sup _{x \in \mathbb{R}}\left|P\left(\frac{W_{K_{1}, K_{2}}-\theta E K_{1} E K_{2}}{\sqrt{\operatorname{Var}^{*}\left(\widehat{W}_{K_{1}, K_{2}}\right)}} \leqslant x\right)-\Phi(x)\right| \\
\leqslant \frac{11.2}{\sqrt{n}}\left\{\frac{a_{2} b_{1} \tau}{a_{1}}+\frac{b_{2}}{a_{2}}\right\} \\
+\frac{3.1}{\sqrt{n}} \frac{\sqrt{\mu}\left(\sqrt{\tau / a_{1}}+\sqrt{a_{1} / a_{2}^{2} \tau}\right)}{\sqrt{\sigma_{1}^{2}+\sigma_{2}^{2} a_{1} \tau / a_{2}}} \\
+\frac{11.9}{\sqrt{n}} \frac{\gamma_{1} / \sqrt{a_{1} \tau}+\gamma_{2}\left(a_{1} \tau\right)^{3 / 2} / a_{2}^{2}}{\left(\sigma_{1}^{2}+\sigma_{2}^{2} a_{1} \tau / a_{2}\right)^{3 / 2}} \\
+\frac{1}{\sqrt{n}}\left\{\frac{2 b_{2}}{a_{2}}+\frac{6.1 E\left|\xi_{1}\right|^{3}}{\sqrt{\tau}\left(E \xi_{1}^{2}\right)^{3 / 2}}\right\} \\
+\frac{\sigma_{1}}{\theta \sqrt{n}}\left(\frac{1+\sqrt{2}}{\sqrt{a_{1} \tau}}+\frac{3 b_{2} \sqrt{a_{1}}}{a_{2} b_{1}}\right.
\end{aligned}
$$

$$
\begin{gathered}
\left.+\frac{2 b_{2}^{2} \sqrt{a_{1}}}{a_{2}^{2} b_{1} \sqrt{n}}+\frac{2 b_{1}}{a_{1} \sqrt{\tau}}\right) \\
+\frac{\sigma_{2}}{\theta \sqrt{n}}\left\{\frac{16 E\left|\xi_{1}\right|^{3}}{a_{1} b_{1}^{2} \sqrt{a_{2} m}}+\frac{2}{\sqrt{a_{2}}}+\frac{2 a_{1} b_{2}}{a_{2} b_{1} \sqrt{a_{2} \tau}}\right\} \\
+\frac{\sigma_{2}}{\theta \sqrt{n}}\left(\frac{2 b_{1}}{a_{1} \sqrt{a_{2} n}}+\frac{2 \sqrt{2}}{b_{1} \sqrt{a_{2}}}\right. \\
\left.+\frac{4 \sqrt{2} b_{1}^{2}}{m a_{1}^{2} \sqrt{a_{2}}}+\frac{2 \sqrt{2}}{m a_{1} \sqrt{a_{2}}}\right) \\
+\frac{1}{\sqrt{n}}\left\{\frac{6.1 E\left|\zeta_{1}\right|^{3}}{b_{2}^{3}}+\frac{2 b_{1}}{a_{1} \sqrt{\tau}}\right\}
\end{gathered}
$$

where $\tau=m / n$.

Remark 2 The random variables $K_{1}=\sum_{j=1}^{m} \xi_{j}$ and $K_{2}=\sum_{\ell=1}^{n} \zeta_{\ell}$ depend on the parameters $m$ and $n$, respectively. It is easy to see that $K_{1}, K_{2}$ satisfy the conditions (C1), (C3) and (C4) in Theorem 3. Moreover, if $m / n$ converges to some constant when $m$ and $n$ tend to infinity, then $K_{1}, K_{2}$ satisfy the condition (C2). Hence the rate of convergence is $O\left(n^{-1 / 2}\right)$.

\section{APPLICATION IN LROC ANALYSIS}

A location receiver operating characteristic (LROC) curve, introduced by Starr, Metz, Lusted and Goodenoughet ${ }^{13}$, provides a useful method in radiology based on a location of data. And the area under the LROC curve has been widely used to measure the diagnostic accuracy of imaging study because it represents the probability that positive and negative case are correctly classified. By using an empirical estimation model, Tang and Balakrishnan ${ }^{10}$ showed that the area under the LROC curve is equivalent to the random-sum Wilcoxon's statistic as the following:

Let $K$ be a binomial distributed random variables with parameters $m$ and $p$. Suppose that the $X_{i}$ 's, $Y_{j}$ 's and $K$ are all independent. An estimator for the area under the LROC curve is given by

$$
A_{\mathrm{LROC}}=\frac{1}{m} \frac{1}{n} \sum_{i=1}^{K} \sum_{j=1}^{n} I\left(X_{i} \geqslant Y_{j}\right) .
$$

Tang and Balakrishnan ${ }^{10}$ also obtained the asymptotic distribution of $A_{\mathrm{LROC}}$ as the following statement.

Theorem 6 (Ref. 10) Let $n$ be any fixed-index and $K$ be a binomial distributed random variable with parameters $m$ and $p$. Suppose that $m / n \rightarrow \tau$ for some constant $\tau>0$ as $m \rightarrow \infty$ and $n \rightarrow \infty$. Under 
the null hypothesis that the distributions of $X$ and $Y$ are equal, we have

$$
\left|P\left(\frac{\sqrt{n}\left(A_{\mathrm{LROC}}-p / 2\right)}{m \sqrt{p(4 n-3 n p+m p) / 12}} \leqslant x\right)-\Phi(x)\right|
$$

tends to zero as $m \rightarrow \infty$ and $n \rightarrow \infty$.

In the next theorem, we investigate Berry-Esseen bounds for $A_{\mathrm{LROC}}$.

Theorem 7 Let $n$ be any fixed-index and $K$ be a binomial distributed random variable with parameters $m$ and $p$. Set $\tau=m / n$. Suppose that the $X_{i}$ 's, $Y_{j}$ 's and $K$ are independent. Under the null hypothesis that the distributions of $X$ and $Y$ are equal, we have the following results:

$$
\begin{gathered}
\sup _{x \in \mathbb{R}}\left|P\left(\frac{A_{\mathrm{LROC}}-K / 2 m}{m n \sqrt{n K(n+K) / 12}} \leqslant x\right)-\Phi(x)\right| \\
\leqslant \frac{11.2}{\sqrt{n}} \frac{\sqrt{1-p}}{\sqrt{p \tau}}+\frac{5.4}{\sqrt{n}} \frac{\sqrt{1 / p \tau}+\sqrt{p \tau}}{\sqrt{1+p \tau}} \\
+\frac{15.5}{\sqrt{n}} \frac{\sqrt{1 / p \tau}+(1 / p \tau)^{3 / 2}}{(1+p \tau)^{3 / 2}},
\end{gathered}
$$

and there exists an absolute constant $C$ (which does not depend on $m$, $n$ and $p$ ) such that for all $x \in \mathbb{R}$,

$$
\begin{array}{r}
\left|P\left(\frac{A_{\mathrm{LROC}}-K / 2 m}{m n \sqrt{n K(n+K) / 12}} \leqslant x\right)-\Phi(x)\right| \\
\leqslant \frac{C}{(1+x)^{2} n}\left\{\frac{1-p}{p \tau}+\frac{(\sqrt{1 / p \tau}+\sqrt{p \tau})^{2}}{1+p \tau}\right\} \\
+\frac{C}{(1+x)^{3} \sqrt{n}}\left(\frac{\sqrt{1 / p \tau}+\sqrt{p \tau}}{\sqrt{1+p \tau}}\right. \\
\left.+\frac{\sqrt{1 / p \tau}+(1 / p \tau)^{3 / 2}}{(1+p \tau)^{3 / 2}}\right) .
\end{array}
$$

Proof: Under the assumption that the distributions of $X$ and $Y$ are equal, Alberink ${ }^{4}$ showed that $\theta=1 / 2$, $\sigma_{1}^{2}=\sigma_{2}^{2}=1 / 12$, and $\gamma_{1}=\gamma_{2}=1 / 32$. From these facts, the bounds in Theorem 4 can be simplified to Theorem 7.

\section{PROOF OF MAIN RESULTS}

In the proof of Theorem 4, we apply Theorem 2 (Berry-Esseen theorem for Mann-Whitney's statistics) to investigate the bounds for the randomly-indexed summands. In the proof of Theorem 5, we obtain the bounds for random summands with non-random centring and non-random norming by improving the arguments of Chen, Goldstein and Shao (see p. 271 of Ref. 14) for the case of two random indices.

Proof of Theorem 4: (Uniform bound.) By using the conditional probability given by $K_{1}, K_{2}$ and applying the uniform bound of Theorem 2 , we can see that

$$
\begin{aligned}
& \left|P\left(\frac{W_{K_{1}, K_{2}}-\theta K_{1} K_{2}}{\sqrt{\sigma_{1}^{2} K_{1} K_{2}^{2}+\sigma_{2}^{2} K_{2} K_{1}^{2}}} \leqslant x\right)-\Phi(x)\right| \\
& \leqslant P\left(\left|K_{1}-E K_{1}\right|>0.09 E K_{1}\right) \\
& +\quad \sum P\left(K_{1}=k_{1}\right) \\
& \left\{0.91 E K_{1} \leqslant k_{1} \leqslant 1.01 E K_{1}\right\} \\
& \times\left|P\left(\frac{W_{k_{1}, K_{2}}-k_{1} \theta K_{2}}{\sqrt{k_{1} \sigma_{1}^{2} K_{2}^{2}+k_{1}^{2} \sigma_{2}^{2} K_{2}}} \leqslant x\right)-\Phi(x)\right| \\
& \leqslant P\left(\left|K_{1}-E K_{1}\right|>0.09 E K_{1}\right) \\
& +P\left(\left|K_{2}-E K_{2}\right|>0.09 E K_{2}\right) \\
& +\sum_{\substack{\left\{0.91 E K_{1} \leqslant k_{1} \leqslant 1.09 E K_{1}\right\} \\
\left\{0.91 E K_{2} \leqslant k_{2} \leqslant 1.09 E K_{2}\right\}}} P\left(K_{1}=k_{1}\right) P\left(K_{2}=k_{2}\right) \\
& \times\left|P\left(\frac{W_{k_{1}, k_{2}}-k_{1} k_{2} \theta}{\sqrt{k_{1} k_{2}^{2} \sigma_{1}^{2}+k_{2} k_{1}^{2} \sigma_{2}^{2}}} \leqslant x\right)-\Phi(x)\right| \\
& \leqslant 11.2\left\{\frac{\sqrt{\operatorname{Var}\left(K_{1}\right)}}{E K_{1}}+\frac{\sqrt{\operatorname{Var}\left(K_{2}\right)}}{E K_{2}}\right\} \\
& +\sum_{\substack{\left\{0.91 E K_{1} \leqslant k_{1} \leqslant 1.09 E K_{1}\right\} \\
\left\{0.91 E K_{2} \leqslant k_{2} \leqslant 1.09 E K_{2}\right\}}} P\left(K_{1}=k_{1}\right) P\left(K_{2}=k_{2}\right) \\
& \times \frac{1}{\sqrt{k}_{2}}\left\{\frac{(1+\sqrt{2}) \sqrt{\mu}\left(\sqrt{k_{2} / k_{1}}+\sqrt{k_{1} / k_{2}}\right)}{\sqrt{\sigma_{1}^{2}+\sigma_{2}^{2} k_{1} / k_{2}}}\right. \\
& \left.+\frac{6.6\left(\gamma_{1} \sqrt{k_{2} / k_{1}}+\gamma_{2}\left(k_{1} / k_{2}\right)^{3 / 2}\right)}{\left(\sigma_{1}^{2}+k_{1} \sigma_{2}^{2} / k_{2}\right)^{3 / 2}}\right\} \\
& \leqslant \frac{11.2}{\sqrt{E K_{2}}}\left\{\sqrt{\frac{\operatorname{Var}\left(K_{1}\right)}{E K_{1}}} \sqrt{\frac{E K_{2}}{E K_{1}}}+\sqrt{\frac{\operatorname{Var}\left(K_{2}\right)}{E K_{2}}}\right\} \\
& +\frac{3.1 \sqrt{\mu}\left(\sqrt{E K_{2} / E K_{1}}+\sqrt{E K_{1} / E K_{2}}\right)}{\sqrt{E K_{2}} \sqrt{\sigma_{1}^{2}+\sigma_{2}^{2} E K_{1} / E K_{2}}} \\
& +\frac{11.9\left\{\gamma_{1} \sqrt{E K_{2} / E K_{1}}+\gamma_{2}\left(E K_{1} / E K_{2}\right)^{3 / 2}\right\}}{\sqrt{E K_{2}}\left(\sigma_{1}^{2}+\sigma_{2}^{2} E K_{1} / E K_{2}\right)^{3 / 2}},
\end{aligned}
$$

where we used the facts that for $\ell=1,2$,

$$
0.91 E K_{\ell} \leqslant k_{\ell} \leqslant 1.09 E K_{\ell}
$$


and that

$$
\left(\sigma_{1}^{2}+\sigma_{2}^{2} E K_{1} / E K_{2}\right) \leqslant \frac{109}{91}\left(\sigma_{1}^{2}+k_{1} \sigma_{2}^{2} / k_{2}\right)
$$

in the last inequality.

(Non-uniform bound.) For all $k_{1}, k_{2} \in \mathbb{N}$, put $p_{\bar{k}}=P\left(K_{1}=k_{1}\right) P\left(K_{2}=k_{2}\right)$ where $\bar{k}=\left(k_{1}, k_{2}\right)$ and set

$$
\overline{\mathrm{A}}:=\left\{\left(k_{1}, k_{2}\right) \in \mathbb{N} \times \mathbb{N} \mid \begin{array}{l|l}
0.5 E K_{1} \leqslant k_{1} \leqslant 1.5 E K_{1} \\
0.5 E K_{2} \leqslant k_{2} \leqslant 1.5 E K_{2}
\end{array}\right\} .
$$

From (1), it suffices to investigate the non-uniform bound for $|x|>1$. Without loss of generality, we may assume that $x>1$.

By using the non-uniform bound of Theorem 2 and taking the conditional probability given by $K_{1}$ and $K_{2}$, we have

$$
\begin{aligned}
& \left|P\left(\frac{W_{K_{1}, K_{2}}-\theta K_{1} K_{2}}{\sqrt{\sigma_{1}^{2} K_{1} K_{2}^{2}+\sigma_{2}^{2} K_{2} K_{1}^{2}}} \leqslant x\right)-\Phi(x)\right| \\
& \leqslant \sum_{\bar{k} \notin \overline{\mathrm{A}}} p_{\bar{k}}\left|P\left(\frac{W_{k_{1}, k_{2}}-k_{1} k_{2} \theta}{\sqrt{k_{1} k_{2}^{2} \sigma_{1}^{2}+k_{2} k_{1}^{2} \sigma_{2}^{2}}} \leqslant x\right)-\Phi(x)\right| \\
& +\frac{C \mu}{(1+x)^{2}} \sum_{\bar{k} \in \overline{\mathrm{A}}} p_{\bar{k}} \frac{\left(\sqrt{k_{2} / k_{1}}+\sqrt{k_{1} / k_{2}}\right)^{2}}{k_{2}\left(\sigma_{1}^{2}+\sigma_{2}^{2} k_{1} / k_{2}\right)} \\
& +\frac{C \sqrt{\mu}}{(1+x)^{3}} \sum_{\bar{k} \in \overline{\mathrm{A}}} p_{\bar{k}} \frac{\sqrt{k_{2} / k_{1}}+\sqrt{k_{1} / k_{2}}}{\sqrt{k_{2}} \sqrt{\sigma_{1}^{2}+\sigma_{2}^{2} k_{1} / k_{2}}} \\
& +\frac{C}{(1+x)^{3}} \sum_{\bar{k} \in \overline{\mathrm{A}}} p_{\bar{k}} \frac{\gamma_{1} \sqrt{k_{2} / k_{1}}+\gamma_{2}\left(k_{1} / k_{2}\right)^{3 / 2}}{\sqrt{k_{2}}\left(\sigma_{1}^{2}+\sigma_{2}^{2} k_{2} / k_{1}\right)^{3 / 2}} \\
& \leqslant \sum_{\bar{k} \notin \overline{\mathrm{A}}} p_{\bar{k}}\left|P\left(\frac{W_{k_{1}, k_{2}}-k_{1} k_{2} \theta}{\sqrt{k_{1} k_{2}^{2} \sigma_{1}^{2}+k_{2} k_{1}^{2} \sigma_{2}^{2}}} \leqslant x\right)-\Phi(x)\right| \\
& +\frac{C \mu\left(\sqrt{E K_{2} / E K_{1}}+\sqrt{E K_{1} / E K_{2}}\right)^{2}}{(1+x)^{2} E K_{2}\left(\sigma_{1}^{2}+\sigma_{2}^{2} E K_{1} / E K_{2}\right)} \\
& +\frac{C \sqrt{\mu}\left(\sqrt{E K_{2} / E K_{1}}+\sqrt{E K_{1} / E K_{2}}\right)}{(1+x)^{3} \sqrt{E K_{2}} \sqrt{\sigma_{1}^{2}+\sigma_{2}^{2} E K_{1} / E K_{2}}} \\
& +\frac{C\left\{\gamma_{1} \sqrt{E K_{2} / E K_{1}}+\gamma_{2}\left(E K_{1} / E K_{2}\right)^{3 / 2}\right\}}{(1+x)^{3} \sqrt{E K_{2}}\left(\sigma_{1}^{2}+\sigma_{2}^{2} E K_{1} / E K_{2}\right)^{3 / 2}} .
\end{aligned}
$$

Set

$$
\widehat{W}_{\bar{k}}:=\sum_{i=1}^{k_{1}} \mathrm{~g}_{1}\left(X_{i}\right)+\sum_{j=1}^{k_{2}} \mathrm{~g}_{2}\left(Y_{j}\right),
$$

where

$$
\begin{aligned}
\mathrm{g}_{1}\left(X_{i}\right) & :=E\left(W_{k_{1}, k_{2}}-k_{1} k_{2} \theta \mid X_{i}\right) \\
& =k_{2}\left\{E\left\{I(X>Y) \mid X=X_{i}\right\}-\theta\right\},
\end{aligned}
$$

and

$$
\begin{aligned}
\mathrm{g}_{2}\left(Y_{j}\right) & :=E\left(W_{k_{1}, k_{2}}-k_{1} k_{2} \theta \mid Y_{j}\right) \\
& =k_{1}\left\{E\left\{I(X>Y) \mid Y=Y_{j}\right\}-\theta\right\} .
\end{aligned}
$$

Note that $E\left(\widehat{W}_{\bar{k}}\right)=0$ and

$$
\operatorname{Var}\left(\widehat{W}_{\bar{k}}\right)=k_{1} k_{2}^{2} \sigma_{1}^{2}+k_{2} k_{1}^{2} \sigma_{2}^{2} .
$$

Set

$$
\Delta_{\bar{k}}:=W_{k_{1}, k_{2}}-k_{1} k_{2} \theta-\widehat{W}_{\bar{k}} .
$$

Hence we can check that

$\Delta_{\bar{k}}=\sum_{i=1}^{k_{1}} \sum_{j=1}^{k_{2}}\left\{I\left(X_{j}, Y_{j}\right)-\theta-\frac{\mathrm{g}_{1}\left(X_{i}\right)}{k_{2}}-\frac{\mathrm{g}_{2}\left(Y_{j}\right)}{k_{1}}\right\}$,

and that

$$
E\left|\Delta_{\bar{k}}\right|^{2}=k_{1} k_{2}\left\{\mu-\sigma_{1}^{2}-\sigma_{2}^{2}\right\} .
$$

Observe that

$$
\begin{aligned}
&\left|P\left(\frac{W_{k_{1}, k_{2}}-k_{1} k_{2} \theta}{\sqrt{k_{1} k_{2}^{2} \sigma_{1}^{2}+k_{2} k_{1}^{2} \sigma_{2}^{2}}} \leqslant x\right)-\Phi(x)\right| \\
& \leqslant P\left(\frac{\widehat{W}_{\bar{k}}}{\sqrt{k_{1} k_{2}^{2} \sigma_{1}^{2}+k_{2} k_{1}^{2} \sigma_{2}^{2}}}>\frac{x-2}{3}\right) \\
& \quad+P\left(\frac{\left|\Delta_{\bar{k}}\right|}{\sqrt{k_{1} k_{2}^{2} \sigma_{1}^{2}+k_{2} k_{1}^{2} \sigma_{2}^{2}}}>\frac{1+x}{3}\right) \\
& \quad+|1-\Phi(x)| \\
& \leqslant \frac{C}{(1+x)^{2}}\left\{1+\frac{E\left|\widehat{W}_{\bar{k}}\right|^{2}+E\left|\Delta_{\bar{k}}\right|^{2}}{k_{1} k_{2}\left(k_{2} \sigma_{1}^{2}+k_{1} \sigma_{2}^{2}\right)}\right\} \\
& \leqslant \frac{C}{(1+x)^{2}}\left\{1+\frac{\mu}{k_{2} \sigma_{1}^{2}+k_{1} \sigma_{2}^{2}}\right\} .
\end{aligned}
$$

From (3) and the fact that $\sigma_{\ell}^{2} \leqslant \mu$ for $\ell=1,2$, we can see that

$$
\begin{aligned}
& \sum_{\bar{k} \notin \overline{\mathrm{A}}} p_{\bar{k}}\left|P\left(\frac{W_{k_{1}, k_{2}}-k_{1} k_{2} \theta}{\sqrt{k_{1} k_{2}^{2} \sigma_{1}^{2}+k_{2} k_{1}^{2} \sigma_{2}^{2}}} \leqslant x\right)-\Phi(x)\right| \\
& \leqslant \frac{C}{(1+x)^{2}} \sum_{\left\{\left|k_{1}-E K_{1}\right|>0.5 E K_{1}\right\}} P\left(K_{1}=k_{1}\right)\left(1+\frac{\mu}{k_{1} \sigma_{2}^{2}}\right) \\
& \quad+\frac{C}{(1+x)_{\left\{\mid k_{2}\right.}^{2}} \sum_{\left.E K_{2} \mid>0.5 E K_{2}\right\}} P\left(K_{2}=k_{2}\right)\left(1+\frac{\mu}{k_{2} \sigma_{1}^{2}}\right) \\
& \leqslant \frac{C \mu}{(1+x)^{2} \sigma_{2}^{2}} P\left(\left|K_{1}-E K_{1}\right|>0.5 E K_{1}\right) \\
& \quad+\frac{C \mu}{(1+x)^{2} \sigma_{1}^{2}} P\left(\left|K_{2}-E K_{2}\right|>0.5 E K_{2}\right)
\end{aligned}
$$




$$
\leqslant \frac{C \mu}{(1+x)^{2} E K_{2}}\left\{\frac{\operatorname{Var}\left(K_{1}\right)}{\sigma_{2}^{2} E K_{1}} \frac{E K_{2}}{E K_{1}}+\frac{\operatorname{Var}\left(K_{2}\right)}{\sigma_{1}^{2} E K_{2}}\right\} .
$$

Hence, combining (2) and (4), one can prove the nonuniform bound of Theorem 4 .

Proof of Theorem 5: Let $Z_{1}, Z_{2}$ and $Z_{3}$ be independent standard normal random variables which are independent of the $X_{i}$ 's, $Y_{j}$ 's, $K_{1}, K_{2}$. Set

$$
\begin{aligned}
T & :=\frac{W_{K_{1}, K_{2}}-\theta E K_{1} E K_{2}}{\sqrt{\operatorname{Var}^{*}\left(\widehat{W}_{K_{1}, K_{2}}\right)}}, \\
T_{1} & :=\frac{\theta\left(K_{1} K_{2}-E K_{1} E K_{2}\right)+Z_{1} Q}{\sqrt{\operatorname{Var}^{*}\left(\widehat{W}_{K_{1}, K_{2}}\right)}}, \\
T_{2} & :=\frac{\theta\left(K_{2}-E K_{2}\right) E K_{1}+Z_{2} \theta K_{2} \sqrt{\operatorname{Var}\left(K_{1}\right)}+Z_{1} q}{\sqrt{\operatorname{Var}^{*}\left(\widehat{W}_{K_{1}, K_{2}}\right)}}, \\
T_{3} & :=\frac{Z_{3} \theta E K_{1} \sqrt{\operatorname{Var}\left(K_{2}\right)}+Z_{2} \theta E K_{2} \sqrt{\operatorname{Var}\left(K_{1}\right)}+Z_{1} q}{\sqrt{\operatorname{Var}^{*}\left(\widehat{W}_{K_{1}, K_{2}}\right)}},
\end{aligned}
$$

where

$$
\begin{aligned}
Q & :=\sqrt{\sigma_{1}^{2} K_{1} K_{2}^{2}+\sigma_{2}^{2} K_{2} K_{1}^{2}}, \\
q & :=\sqrt{\sigma_{1}^{2} E K_{1} E K_{2}^{2}+\sigma_{2}^{2} E K_{2} E K_{1}^{2}} .
\end{aligned}
$$

It is clear that $T_{3}$ has the standard normal distribution and easy to see that

$$
\begin{aligned}
& \left|P\left(\frac{W_{K_{1}, K_{2}}-\theta E K_{1} E K_{2}}{\left.\sqrt{\operatorname{Var}^{*}\left(\widehat{W}_{K_{1}, K_{2}}\right)} \leqslant x\right)}\right)-\Phi(x)\right| \\
& \leqslant\left|P(T \leqslant x)-P\left(T_{1} \leqslant x\right)\right| \\
& \quad+\left|P\left(T_{1} \leqslant x\right)-P\left(T_{2} \leqslant x\right)\right| \\
& \quad+\left|P\left(T_{2} \leqslant x\right)-P\left(T_{3} \leqslant x\right)\right| \\
& =\left|P\left(\frac{W_{K_{1}, K_{2}}-\theta K_{1} K_{2}}{Q} \leqslant y_{1}\right)-\Phi\left(y_{1}\right)\right| \\
& \quad+\mid P\left(\frac{K_{1}-E K_{1}}{\sqrt{\operatorname{Var}\left(K_{1}\right)}}\right) \\
& \left.\quad+\frac{Z_{1}(Q-q)}{\theta K_{2} \sqrt{\operatorname{Var}\left(K_{1}\right)} \leqslant y_{2}}\right)-\Phi\left(y_{2}\right) \mid \\
& +\mid P\left(\frac{K_{2}-E K_{2}}{\sqrt{\operatorname{Var}\left(K_{2}\right)}}\right. \\
& \left.\quad+\frac{Z_{2}\left(K_{2}-E K_{2}\right) \sqrt{\operatorname{Var}\left(K_{1}\right)}}{E K_{1} \sqrt{\operatorname{Var}\left(K_{2}\right)}} \leqslant y_{3}\right)-\Phi\left(y_{3}\right) \mid \\
& =: R_{1}+R_{2}+R_{3},
\end{aligned}
$$

where $y_{1}, y_{2}$ and $y_{3}$ are given by

$$
y_{1}:=\frac{x \sqrt{\operatorname{Var}^{*}\left(\widehat{W}_{K_{1}, K_{2}}\right)}-\theta\left(K_{1} K_{2}-E K_{1} E K_{2}\right)}{Q},
$$

$y_{2}:=\frac{x \sqrt{\operatorname{Var}^{*}\left(\widehat{W}_{K_{1}, K_{2}}\right)}-\theta\left(K_{2}-E K_{2}\right) E K_{1}-Z_{1} q}{\theta K_{2} \sqrt{\operatorname{Var}\left(K_{1}\right)}}$,

$y_{3}:=\frac{x \sqrt{\operatorname{Var}\left(\widehat{W}_{\mathrm{r}}\right)}-Z_{2} \theta E K_{2} \sqrt{\operatorname{Var}\left(K_{1}\right)}-Z_{1} q}{\theta E K_{1} \sqrt{\operatorname{Var}\left(K_{2}\right)}}$.

Firstly, following the arguments of (1) (in the proof of Theorem 4), we can see that

$$
\begin{aligned}
R_{1} \leqslant & \frac{11.2}{\sqrt{E K_{2}}}\left\{\sqrt{\frac{\operatorname{Var}\left(K_{1}\right)}{E K_{1}}} \sqrt{\frac{E K_{2}}{E K_{1}}}+\sqrt{\frac{\operatorname{Var}\left(K_{2}\right)}{E K_{2}}}\right\} \\
& +\frac{3.1 \sqrt{\mu}\left(\sqrt{E K_{2} / E K_{1}}+\sqrt{E K_{1} / E K_{2}}\right)}{\sqrt{E K_{2}} \sqrt{\sigma_{1}^{2}+\sigma_{2}^{2} E K_{1} / E K_{2}}} \\
& +\frac{11.9\left\{\gamma_{1} \sqrt{E K_{2} / E K_{1}}+\gamma_{2}\left(E K_{1} / E K_{2}\right)^{3 / 2}\right\}}{\sqrt{E K_{2}}\left(\sigma_{1}^{2}+\sigma_{2}^{2} E K_{1} / E K_{2}\right)^{3 / 2}} \\
= & \frac{11.2}{\sqrt{n}}\left\{\frac{a_{2} b_{1} \tau}{a_{1}}+\frac{b_{2}}{a_{2}}\right\} \\
& +\frac{3.1}{\sqrt{n}} \frac{\sqrt{\mu}\left(\sqrt{\tau / a_{1}}+\sqrt{a_{1} / a_{2}^{2} \tau}\right)}{\sqrt{\sigma_{1}^{2}+\sigma_{2}^{2} a_{1} \tau / a_{2}}} \\
& +\frac{11.9}{\sqrt{n}} \frac{\gamma_{1} / \sqrt{a_{1} \tau}+\gamma_{2}\left(a_{1} \tau\right)^{3 / 2} / a_{2}^{2}}{\left(\sigma_{1}^{2}+\sigma_{2}^{2} a_{1} \tau / a_{2}\right)^{3 / 2}} .
\end{aligned}
$$

Secondly, using the conditional probability given by $K_{2}$ and $Z_{1}$,

$$
\begin{aligned}
R_{2} \leqslant P & \left(\left|K_{2}-E K_{2}\right|>0.5 E K_{2}\right) \\
& +\sum_{\substack{\left\{0.5 E K_{2} \leqslant k_{2} \leqslant 1.5 E K_{2}\right\} \\
\quad}} P\left(K_{2}=k_{2}\right) \\
& \quad \times\left(S_{1}+\Delta \leqslant y_{2}\right)-\Phi\left(y_{2}\right) \mid,
\end{aligned}
$$

where

$$
S_{1}=\frac{K_{1}-E K_{1}}{\sqrt{\operatorname{Var}\left(K_{1}\right)}}
$$

and

$$
\Delta=\frac{Z_{1}\left(\sqrt{k_{2}^{2} \sigma_{1}^{2} K_{1}+k_{2} \sigma_{2}^{2} K_{1}^{2}}-q\right)}{k_{2} \theta \sqrt{\operatorname{Var}\left(K_{1}\right)}} .
$$

For each $i=1, \ldots, m$, let

$$
\Delta_{i}=\frac{Z_{1}\left(\sqrt{k_{2}^{2} \sigma_{1}^{2} \epsilon_{i}+k_{2} \sigma_{2}^{2} \epsilon_{i}^{2}}-q\right)}{k_{2} \theta \sqrt{\operatorname{Var}\left(K_{1}\right)}},
$$


where $\epsilon_{i}=K_{1}-\xi_{i}+a_{1}$. Note that

$$
\begin{aligned}
& E\left|S_{1} \Delta\right| \\
& \leqslant \frac{E\left|S_{1}\left\{k_{2}^{2} \sigma_{1}^{2} K_{1}+k_{2} \sigma_{2}^{2} K_{1}^{2}-q^{2}\right\}\right|}{q k_{2} \theta \sqrt{\operatorname{Var}\left(K_{1}\right)}} \\
& \leqslant \frac{\sigma_{1}^{2} E\left|S_{1}\left\{k_{2}^{2}\left(K_{1}-E K_{1}\right)+k_{2}\left(k_{2}-E K_{2}\right) E K_{1}\right\}\right|}{q k_{2} \theta \sqrt{\operatorname{Var}\left(K_{1}\right)}} \\
& +\frac{\sigma_{1}^{2} E\left|S_{1}\left\{\left(k_{2}-E K_{2}\right) E K_{1} E K_{2}+\left(E^{2} K_{2}-E K_{2}^{2}\right) E K_{1}\right\}\right|}{q k_{2} \theta \sqrt{\operatorname{Var}\left(K_{1}\right)}} \\
& +\frac{\sigma_{2}^{2} E\left|S_{1}\left\{k_{2}\left(K_{1}-E K_{1}\right)^{2}+2 k_{2}\left(K_{1}-E K_{1}\right) E K_{1}\right\}\right|}{q k_{2} \theta \sqrt{\operatorname{Var}\left(K_{1}\right)}} \\
& +\frac{\sigma_{2}^{2} E\left|S_{1}\left\{\left(k_{2}-E K_{2}\right) E^{2} K_{1}+\left(E^{2} K_{1}-E K_{1}^{2}\right) E K_{2}\right\}\right|}{q k_{2} \theta \sqrt{\operatorname{Var}\left(K_{1}\right)}} \\
& \leqslant \frac{\sigma_{1}\left\{k_{2}^{2} \sqrt{\operatorname{Var}\left(K_{1}\right)}+k_{2}\left|k_{2}-E K_{2}\right| E K_{1}\right\}}{k_{2} \theta E K_{2} \sqrt{E K_{1}} \sqrt{\operatorname{Var}\left(K_{1}\right)}} \\
& +\frac{\sigma_{1}\left\{\left|k_{2}-E K_{2}\right| E K_{1} E K_{2}+E K_{1} \operatorname{Var}\left(K_{2}\right)\right\}}{k_{2} \theta E K_{2} \sqrt{E K_{1}} \sqrt{\operatorname{Var}\left(K_{1}\right)}} \\
& +\frac{\sigma_{2}\left\{k_{2} E\left|K_{1}-E K_{1}\right|^{3}+2 k_{2} E K_{1} \sqrt{\operatorname{Var}\left(K_{1}\right)}\right\}}{k_{2} \theta E K_{1} \sqrt{E K_{2}} \sqrt{\operatorname{Var}\left(K_{1}\right)}} \\
& +\frac{\sigma_{2}\left\{\left|k_{2}-E K_{2}\right| E^{2} K_{1}+E K_{2} \operatorname{Var}\left(K_{1}\right)\right\}}{k_{2} \theta E K_{1} \sqrt{E K_{2}} \sqrt{\operatorname{Var}\left(K_{1}\right)}} \\
& \leqslant \frac{\sigma_{1}}{\theta}\left\{\frac{k_{2}}{a_{2} n \sqrt{a_{1} m}}+\frac{\left|k_{2}-E K_{2}\right| \sqrt{a_{1}}}{a_{2} n b_{1}}\right\} \\
& +\frac{\sigma_{1}}{\theta}\left\{\frac{\left|k_{2}-E K_{2}\right| \sqrt{a_{1}}}{k_{2} b_{1}}+\frac{b_{2}^{2} \sqrt{a_{1}}}{k_{2} a_{2} b_{1}}\right\} \\
& +\frac{\sigma_{2}}{\theta}\left\{\frac{16 E\left|\xi_{1}\right|^{3}}{a_{1} b_{1}^{2} \sqrt{a_{2} m n}}+\frac{2}{\sqrt{a_{2} n}}\right\} \\
& +\frac{\sigma_{2}}{\theta}\left\{\frac{\left|k_{2}-E K_{2}\right| a_{1} \sqrt{m}}{k_{2} b_{1} \sqrt{a_{2} n}}+\frac{b_{1} \sqrt{a_{2}}}{k_{2} a_{1}}\right\},
\end{aligned}
$$

where we used the fact that (Rosenthal's inequality)

$$
\begin{aligned}
E \mid K_{1}- & \left.E K_{1}\right|^{3} \\
& \leqslant 8\left\{\sum_{i=1}^{m} E\left|\xi_{i}\right|^{3}+\left(\sum_{i=1}^{m} E \xi_{i}^{2}\right)^{3 / 2}\right\}
\end{aligned}
$$

in the last inequality.

Also, we can see that

$$
\begin{aligned}
& E\left|\left(\xi_{i}-a_{1}\right)\left(\Delta-\Delta_{i}\right)\right| \\
& \leqslant E\left\{\frac{\left|\xi_{i}-a_{1}\right|\left|k_{2}^{2} \sigma_{1}^{2}\left(\xi_{i}-a_{1}\right)+k_{2} \sigma_{2}^{2}\left\{K_{1}^{2}-\epsilon_{i}^{2}\right\}\right|}{\left.k_{2} \theta \sqrt{\operatorname{Var}\left(K_{1}\right)} \sqrt{k_{2}^{2} \sigma_{1}^{2} \epsilon_{i}+k_{2} \sigma_{2}^{2} \epsilon_{i}^{2}}\right\}}\right. \\
& \leqslant E\left\{\frac{\sigma_{1}\left(\xi_{i}-a_{1}\right)^{2}}{\left.\theta \sqrt{\epsilon_{i}} \sqrt{\operatorname{Var}\left(K_{1}\right)}\right\}}\right. \\
& +E\left\{\frac{\sigma_{2}\left|\xi_{i}-a_{1}\right|\left|2 \epsilon_{i}+\left(\xi_{i}-a\right)\right|}{\theta \sqrt{k_{2}}\left|\epsilon_{i}\right| \sqrt{\operatorname{Var}\left(K_{1}\right)}}\right\}
\end{aligned}
$$

$$
\begin{aligned}
& \leqslant E\left\{\frac{\sigma_{1}\left(\xi_{i}-a_{1}\right)^{2}}{\theta \sqrt{\epsilon_{i}} \sqrt{\operatorname{Var}\left(K_{1}\right)}}\right\} \\
& +E\left\{\frac{2 \sigma_{2}\left|\xi_{i}-a_{1}\right|}{\theta \sqrt{k_{2}} \sqrt{\operatorname{Var}\left(K_{1}\right)}}+\frac{\sigma_{2}\left(\xi_{i}-a\right)^{2}}{\theta \sqrt{k_{2}}\left|\epsilon_{i}\right| \sqrt{\operatorname{Var}\left(K_{1}\right)}}\right\} \\
& \leqslant \frac{\sigma_{1} b_{1}}{\theta \sqrt{m}}\left(\frac{2 b_{1}}{a_{1} \sqrt{m}}+\frac{\sqrt{2}}{\sqrt{a_{1} m}}\right) \\
& +\frac{2 \sigma_{2}}{\theta \sqrt{k_{2} m}}+\frac{\sigma_{2} b_{1}}{\theta \sqrt{k_{2} m}}\left(\frac{4 b_{1}^{2}}{a_{1}^{2} m}+\frac{2}{a_{1} m}\right),
\end{aligned}
$$

where we use the fact that

$$
\begin{aligned}
& E \epsilon_{i}^{-1} \\
& \leqslant P\left(\left|\epsilon_{i}-E K_{1}\right|>0.5 E K_{1}\right) \\
& \quad+E\left\{\frac{I\left(\left|\epsilon_{i}-E K_{1}\right| \leqslant 0.5 E K_{1}\right)}{\epsilon_{i}}\right\} \\
& \leqslant \frac{4 \operatorname{Var}\left(K_{1}\right)}{\left(E K_{1}\right)^{2}}+\frac{2}{E K_{1}} \\
& =\frac{4 b_{1}^{2}}{a_{1}^{2} m}+\frac{2}{a_{1} m}
\end{aligned}
$$

in the last inequality.

By applying the result of p. 258 of Ref. 14 (the uniform bound for nonlinear statistics), we have that

$$
\begin{aligned}
& \sup _{z \in \mathbb{R}}\left|P\left(S_{1}+\Delta \leqslant z\right)-\Phi(z)\right| \\
& \leqslant \frac{6.1 E|\xi|^{3}}{\sqrt{m}\left(E \xi^{2}\right)^{3 / 2}}+E\left|S_{1} \Delta\right| \\
& +\sum_{i=1}^{m} \frac{E\left|\left(\xi_{i}-a_{1}\right)\left(\Delta-\Delta_{i}\right)\right|}{b_{1} \sqrt{m}} .
\end{aligned}
$$

Hence combining (7)-(10), we obtain

$$
\begin{aligned}
R_{2} \leqslant & P\left(\left|K_{2}-E K_{2}\right| \geqslant 0.5 E K_{2}\right) \\
+ & P\left(K_{2}=k_{2}\right) \\
& \times \mid P\left(S_{1}+\Delta \leqslant K_{2} \leqslant k_{2} \leqslant 1.5 E K_{2}\right\} \\
\leqslant & \frac{2 \sqrt{\operatorname{Var}\left(K_{2}\right)}}{E K_{2}}+\frac{6.1 E|\xi|^{3}}{\sqrt{m}\left(E \xi^{2}\right)^{3 / 2}} \\
+ & \left\{0.5 E K_{2} \leqslant k_{2} \leqslant 1.5 E K_{2}\right\} \\
\times & \left\{\frac{\sigma_{1}}{\theta}\left(\frac{k_{2}}{a_{2} n \sqrt{a_{1} m}}+\frac{\left|k_{2}-E K_{2}\right| \sqrt{a_{1}}}{a_{2} n b_{1}}\right)\right. \\
& +\frac{\sigma_{1}}{\theta}\left(\frac{\left|k_{2}-E K_{2}\right| \sqrt{a_{1}}}{k_{2} b_{1}}+\frac{b_{2}^{2} \sqrt{a_{1}}}{k_{2} a_{2} b_{1}}\right) \\
& +\frac{\sigma_{2}}{\theta}\left(\frac{16 E\left|\xi_{1}\right|^{3}}{a_{1} b_{1}^{2} \sqrt{a_{2} m n}}+\frac{2}{\sqrt{a_{2} n}}\right)
\end{aligned}
$$




$$
\begin{aligned}
& +\frac{\sigma_{2}}{\theta}\left(\frac{\left|k_{2}-E K_{2}\right| a_{1} \sqrt{m}}{k_{2} b_{1} \sqrt{a_{2} n}}+\frac{b_{1} \sqrt{a_{2}}}{k_{2} a_{1}}\right) \\
& +\frac{\sigma_{1}}{\theta}\left(\frac{2 b_{1}}{a_{1} \sqrt{m}}+\frac{\sqrt{2}}{\sqrt{a_{1} m}}\right) \\
& \left.+\frac{2 \sigma_{2}}{\theta b_{1} \sqrt{k_{2}}}+\frac{\sigma_{2}}{\theta \sqrt{k_{2}}}\left(\frac{4 b_{1}^{2}}{a_{1}^{2} m}+\frac{2}{a_{1} m}\right)\right\} \\
& \leqslant \frac{2 \sqrt{\operatorname{Var}\left(K_{2}\right)}}{E K_{2}}+\frac{6.1 E\left|\xi_{1}\right|^{3}}{\sqrt{m}\left(E \xi_{1}^{2}\right)^{3 / 2}} \\
& +\frac{\sigma_{1}}{\theta}\left\{\frac{E K_{2}}{a_{2} n \sqrt{a_{1} m}}+\frac{\sqrt{\operatorname{Var}\left(K_{2}\right)} \sqrt{a_{1}}}{a_{2} n b_{1}}\right\} \\
& +\frac{\sigma_{1}}{\theta}\left\{\frac{2 \sqrt{\operatorname{Var}\left(K_{2}\right)} \sqrt{a_{1}}}{b_{1} E K_{2}}+\frac{2 b_{2}^{2} \sqrt{a_{1}}}{a_{2} b_{1} E K_{2}}\right\} \\
& +\frac{\sigma_{2}}{\theta}\left\{\frac{16 E\left|\xi_{1}\right|^{3}}{a_{1} b_{1}^{2} \sqrt{a_{2} n m}}+\frac{2}{\sqrt{a_{2} n}}\right\} \\
& +\frac{\sigma_{2}}{\theta}\left\{\frac{2 a_{1} \sqrt{m} \sqrt{\operatorname{Var}\left(K_{2}\right)}}{b_{1} \sqrt{a_{2} n} E K_{2}}+\frac{2 b_{1} \sqrt{a_{2}}}{a_{1} E K_{2}}\right\} \\
& +\frac{\sigma_{1}}{\theta}\left\{\frac{2 b_{1}}{a_{1} \sqrt{m}}+\frac{\sqrt{2}}{\sqrt{a_{1} m}}\right\} \\
& +\frac{2 \sqrt{2} \sigma_{2}}{\theta b_{1} \sqrt{E K_{2}}}+\frac{\sqrt{2} \sigma_{2}}{\theta \sqrt{E K_{2}}}\left\{\frac{4 b_{1}^{2}}{a_{1}^{2} m}+\frac{2}{a_{1} m}\right\} \\
& =\frac{1}{\sqrt{n}}\left\{\frac{2 b_{2}}{a_{2}}+\frac{6.1 E\left|\xi_{1}\right|^{3}}{\sqrt{\tau}\left(E \xi_{1}^{2}\right)^{3 / 2}}\right\} \\
& +\frac{\sigma_{1}}{\theta \sqrt{n}}\left\{\frac{1+\sqrt{2}}{\sqrt{a_{1} \tau}}+\frac{3 b_{2} \sqrt{a_{1}}}{a_{2} b_{1}}\right\} \\
& +\frac{\sigma_{1}}{\theta \sqrt{n}}\left\{\frac{2 b_{2}^{2} \sqrt{a_{1}}}{a_{2}^{2} b_{1} \sqrt{n}}+\frac{2 b_{1}}{a_{1} \sqrt{\tau}}\right\} \\
& +\frac{\sigma_{2}}{\theta \sqrt{n}}\left\{\frac{16 E\left|\xi_{1}\right|^{3}}{a_{1} b_{1}^{2} \sqrt{a_{2} m}}+\frac{2}{\sqrt{a_{2}}}\right\} \\
& +\frac{\sigma_{2}}{\theta \sqrt{n}}\left\{\frac{2 a_{1} b_{2}}{a_{2} b_{1} \sqrt{a_{2} \tau}}+\frac{2 b_{1}}{a_{1} \sqrt{a_{2} n}}\right\} \\
& +\frac{\sigma_{2}}{\theta \sqrt{n}}\left\{\frac{2 \sqrt{2}}{b_{1} \sqrt{a_{2}}}+\frac{4 \sqrt{2} b_{1}^{2}}{m a_{1}^{2} \sqrt{a_{2}}}+\frac{2 \sqrt{2}}{m a_{1} \sqrt{a_{2}}}\right\} \text {. }
\end{aligned}
$$

Finally, we denote

$$
S_{2}=\frac{K_{2}-E K_{2}}{\sqrt{\operatorname{Var}\left(K_{2}\right)}}
$$

and set

$$
\Lambda=\frac{\left(K_{2}-E K_{2}\right) \sqrt{\operatorname{Var}\left(K_{1}\right)}}{E K_{1} \sqrt{\operatorname{Var}\left(K_{2}\right)}} Z_{2} .
$$

For each $j=1, \ldots, n$, let

$$
\Lambda_{j}=\frac{\left\{\left(K_{2}-\zeta_{j}+a_{2}\right)-E K_{2}\right\} \sqrt{\operatorname{Var}\left(K_{1}\right)}}{E K_{1} \sqrt{\operatorname{Var}\left(K_{2}\right)}} Z_{2} .
$$

Note that

$$
\begin{aligned}
E\left|S_{2} \Lambda\right| & \leqslant \frac{\sqrt{E S_{2}^{2}} \sqrt{E\left|K_{2}-E K_{2}\right|^{2}} \sqrt{\operatorname{Var}\left(K_{1}\right)}}{E K_{1} \sqrt{\operatorname{Var}\left(K_{2}\right)}} E\left|Z_{2}\right| \\
& \leqslant \frac{b_{1}}{a_{1} \sqrt{m}}
\end{aligned}
$$

and that

$$
\begin{aligned}
& E\left|\left(\zeta_{j}-a_{2}\right)\left(\Lambda-\Lambda_{j}\right)\right| \\
& \leqslant \frac{E\left(\zeta_{j}-a_{2}\right)^{2} \sqrt{\operatorname{Var}\left(K_{1}\right)}}{E K_{1} \sqrt{\operatorname{Var}\left(K_{2}\right)}} E\left|Z_{2}\right| \\
& \leqslant \frac{b_{1} b_{2}}{a_{1} \sqrt{m n}} .
\end{aligned}
$$

By using the conditional probability given by $Z_{2}, Z_{1}$ and applying the result of p. 258 of Ref. 14, we have

$$
\begin{aligned}
R_{3} & =\left|P\left(S_{2}+\Lambda \leqslant y_{3}\right)-\Phi\left(y_{3}\right)\right| \\
& \leqslant \frac{6.1 E\left|\zeta_{1}\right|^{3}}{\sqrt{n}\left(E \zeta_{1}^{2}\right)^{3 / 2}}+E\left|S_{2} \Lambda\right|+\sum_{j=1}^{n} \frac{E\left|\left(\zeta_{j}-a_{2}\right)\left(\Lambda-\Lambda_{j}\right)\right|}{b_{2} \sqrt{n}} \\
& \leqslant \frac{6.1 E\left|\zeta_{1}\right|^{3}}{\sqrt{n}\left(E \zeta_{1}^{2}\right)^{3 / 2}}+\frac{2 b_{1}}{a_{1} \sqrt{m}} \\
& =\frac{1}{\sqrt{n}}\left\{\frac{6.1 E\left|\zeta_{1}\right|^{3}}{\left(E \zeta_{1}^{2}\right)^{3 / 2}}+\frac{2 b_{1}}{a_{1} \sqrt{\tau}}\right\} .
\end{aligned}
$$

We combine (5), (6), (11) and (12) to obtain the main result.

Acknowledgements: The authors thank Professor Kritsana Neammanee for helpful discussions and the Centre of Excellence in Mathematics CHE for financial support.

\section{REFERENCES}

1. Wilcoxon F (1945) Individual comparisons by ranking methods. Biometrics 1, 80-3.

2. Mann HB, Whitney DR (1947) On a test of whether one of two random variables is stochastically larger than the other. Ann Math Stat 18, 50-60.

3. Pestman WR (1998) Mathematical Statistics: An Introduction, De Gruyter.

4. Alberink IB (2000) A Berry-Esseen bound for $U$ statistics in the non-i.i.d. case. $J$ Theor Probab 13, 519-33.

5. Hoeffding W (1948) A class of statistics with asymptotically normal distribution. Ann Math Stat 19, 293-325. 
6. Lehmann EL (1951) Consistency and unbiasedness of certain nonparametric tests. Ann Math Stat 22, 165-79.

7. Grams WF, Serfling RJ (1973) Convergence rates for $U$-statistics and related statistics. Ann Probab 1, 153-60.

8. Chen LHY, Shao QM (2007) Normal approximation for nonlinear statistics using a concentration inequality approach. Bernoulli 13, 581-99.

9. Chakraborty DP (2006) A search model and figure of merit for observer data acquired according to the freeresponse paradigm. Phys Med Biol 51, 3449-62.

10. Tang LL, Balakrishnan N (2011) A random-sum Wilcoxon statistic and its applications to analysis of ROC and LROC data. J Stat Plann Infer 141, 335-44.

11. Hajek J (1968) Asymptotic normality of simple linear rank statistics under alternatives. Ann Math Stat 39, 325-46.

12. Ross S (1995) Stochastic Processes, 2nd edn, Wiley, New York.

13. Starr SJ, Metz CE, Lust edn LB, Goodenough DJ (1975) Visual detection and localization of radiographic images. Radiology 116, 533-8.

14. Chen LHY, Goldstein L, Shao QM (2010) Normal Approximation by Stein's Method, Springer, New York. 